\title{
含氮杂环协助的醛胺缩合反应机理的研究
}

\author{
李哲伟王寒阅蒲敏* 杨作银雷鸣* \\ (北京化工大学化工资源有效利用国家重点实验室 化学学院 计算化学研究所 北京 100029)
}

\begin{abstract}
摘要 采用密度泛函理论(DFT)方法研究了在酸性条件下对带有氮杂原子的伯胺与醛的缩合反应机理. 研究结果表明, 在酸性条件下, 碱性更强的杂环氮比羰基氧更容易被质子化, 杂环氮作为氢质子桥使缩合反应能垒大大降低, 计算得 到的反应能量跨度为 $13.08 \mathrm{kcal} / \mathrm{mol}$. 同时, 将伯胺上含氮杂环上的 $\mathrm{N}$ 原子调变为 $\mathrm{P}, \mathrm{As}$ 原子及改变 $\mathrm{N}$ 原子的位置后缩 合反应能垒升高, 证实了杂环氮原子协助氢转移的重要性, 此理论研究为揭示含氮杂环伯胺与醛的缩合反应机理提供 了新的思路与理论依据.
\end{abstract}

关键词 含氮杂环; 伯胺; 胺醛缩合; 密度泛函理论; 氢转移

\section{Theoretical Study on Nitrogenous Heterocyclic Assisted Aldimine Condensation}

\author{
Li, Zhewei Wang, Qianyue Pu, Min* Yang, Zuoyin Lei, Ming* \\ (State Key Laboratory of Chemical Resource Engineering, College of Chemistry, Institute of Computational Chemistry, \\ Beijing University of Chemical Technology, Beijing 100029, China)
}

\begin{abstract}
Imines and the intermediate methylamine by the aldimine condensation of primary amines with aldehydes have a potential application in the field of pharmacy, life science, catalysis, material science, etc. In this reaction, the hydrogen transfer in the dehydration step normally prefers the pathway via a water bridge in aqueous solution or a directly dehydration in organic solvent. It is a different mechanism for the aldimine condensation of amine owning neighbouring nitrogenous heterocycle. Herein we investigated the mechanism of aldimine condensation of primary amine containing nitrogenous heterocycle with aldehyde in dichloromethane under acidic conditions using density functional theory (DFT) at $\omega \mathrm{B} 97 \mathrm{X}-\mathrm{D} / 6-31+$ $+\mathrm{G}(\mathrm{d}, \mathrm{p})$ level, the calculated results show that compared with specific acid catalysis, the heterocyclic nitrogen with stronger basicity is easier to be protonated than the oxygen of carbonyl group. The whole reaction proceeds two hydrogen transfer steps via nitrogen bridge owning an energy span of $13.08 \mathrm{kcal} / \mathrm{mol}$. The rate-determing step is the second hydrogen transfer step. In each step the heterocyclic nitrogen is a bridge to assist the hydrogen transfer, which could reduce the free energy barrier of the aldimine condensation. It is unfavorable for the reaction pathway via directly hydrogen transfer with a four-membered ring transition state owning a free energy barrier of $32.73 \mathrm{kcal} / \mathrm{mol}$, and the reaction pathway via a water bridge is not located. Meanwhile, the energy barriers increased for systems in which the $\mathrm{N}$ atom in heterocycle of primary amine is replaced by $\mathrm{P} / \mathrm{As}$ atoms. The rate-determining step changes from the second hydrogen transfer step for $\mathrm{N}$ system to the first hydrogen transfer step for As system. The position effect of adjacent nitrogen atom is also investigated. The $\gamma$ position owns the highest reactivity of the aldimine condensation, which implies that the ring strain plays an important role in the aldimine condensation of primary amine containing nitrogenous heterocycle with aldehyde. This theoretical study may provide insights to unveil the nature of aldimine condensation of aldehyde and primary amine owning nitrogeneous heterocycle. Keywords nitrogenous heterocycle; primary amine; aldimine condensation; DFT; hydrogen transfer
\end{abstract}

\section{1 引言}

伯胺与醛反应生成亚胺, 也称希夫碱 ${ }^{[1]}$. 醛胺缩合 反应是一类重要的化学反应. 亚胺及中间体氨基醇在制 药、生命科学、催化、材料科学等领域中具有广泛的应 用 ${ }^{[2 \sim 10]}$. 自 20 世纪 70 年代开始, 关于伯胺与醛反应生 成亚胺的反应机理的研究被大量报道 ${ }^{[11 \sim 15]}$
在中性和酸性条件下，醛胺缩合反应通常经过三个 步骤形成亚胺. 醛与胺反应首先生成两性离子, 接着生 成氨基醇中间体，最后脱水形成亚胺产物(见图 1). 1974 年 Sayer 等 ${ }^{[16]}$ 通过动力学方法研究了水溶液下醛与伯胺 反应生成氨基醇的反应机理，指出伯胺与醛结合首先形 成两性离子后，然后在水或胺等促进下将氢从胺基转移

* E-mail: pumin@mail.buct.edu.cn; leim@mail.buct.edu.cn

Received November 26, 2019; published April 8, 2020.

Supporting information for this article is available free of charge via the Internet at http://sioc-journal.cn.

Project supported by the National Natural Science Foundation of China (No. 21672018), the State Key Laboratory of Physical Chemistry of Solid Surfaces (Xiamen University) (No. 201811) and the Fundamental Research Funds for the Central Universities (No. XK1802-6).

项目受国家自然科学基金(No. 21672018)、厦门大学固体表面物理化学国家重点实验室开放课题(No. 201811)和中央高校基本科研专项资金项目(No. XK1802-6)资助. 
到羰基氧上得到氨基醇产物. 同年, Rosenberg 等 ${ }^{[17]}$ 在实 验基础上推测该反应是两分子水协助的氢转移过程, 并 预测了中间体结构. 1987 年, Williams ${ }^{[18]}$ 首次计算了氨 和甲醛反应生成氨基甲醇的反应机理，计算结果证实了 水分子协助氢转移反应路径比直接通过极性四元环反 应路径更具优势, 并且两分子水通过氢键桥参与氢质子 转移的反应路径的能垒最低, 其过渡态几何形状基本符 合 Rosenberg 的实验预测. 1998 年, Smith 等 ${ }^{[19]}$ 在 G2(MP2, SVP)水平对甲胺与甲醛反应生成亚胺的反应 机理进行了理论研究同样发现当两分子水参与质子转 移时所形成甲醇胺的反应能垒最低. 但在甲醇胺脱去水 形成亚胺的过程中, 即使两分子水协助反应其反应能垒 也达到 $26.73 \mathrm{kcal} / \mathrm{mol}$, 解释了醛与胺在中性条件下反 应生成氨基醇的实验现象. 而在酸性条件下, 过低的 $\mathrm{pH}$ 值会使胺质子化从而导致反应速率降低 ${ }^{[20]}$, 适当的 $\mathrm{pH}$ 值会加速反应 ${ }^{[21]}$. 因此, 在酸催化下, 氢质子先与醛 底物的羰基氧原子结合形成质子化醛, 然后再与胺形成 质子化氨基醇, 再直接或水分子协助脱水形成质子化希 夫碱, 最后通过碱消除氢得到希夫碱 ${ }^{[11,15,22,23]}$. Muñoz 等 ${ }^{[24 ~ 26]}$ 利用密度泛函理论在(B3LYP/6-31+ $\mathrm{G}^{*}$ ) 水平下 计算研究了维生素 B6 类似物的形成机理, 揭示了羟基 协助氢转移的重要作用, 脱水步骤为反应的决速步骤. 在关于磷脂酰乙醇胺和乙醛之间形成希夫碱的研究中, 他们提出了水分子形成氢键桥传递促进氢质子转移 ${ }^{[27]}$, 总的反应能垒降至 $18.88 \mathrm{kcal} / \mathrm{mol}$. 酸催化下胺的衍生 物如氨基脲的类醛胺缩合反应具有类似的反应机理 ${ }^{[28]}$. 可以看出, 在不同的反应条件及底物环境下, 醛胺缩合 反应会呈现不同的反应模式.
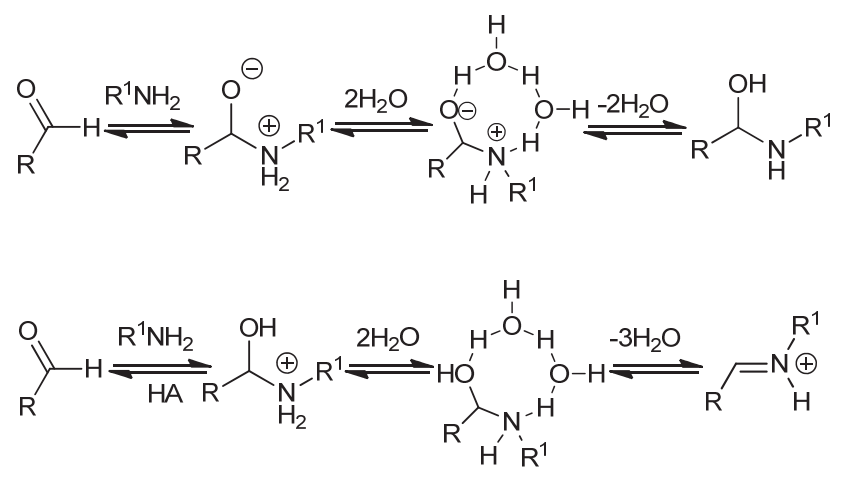

图 1 在中性和酸性水溶液下发生醛胺缩合反应机理示意图

Figure 1 Reaction mechanism of aldimine condensation of amine and aldehyde in neutral and acidic conditions

但是, 多数醛胺缩合反应在水相中进行, 在有机溶 剂中的研究并不是很多 ${ }^{[29]}$. 而且, 该反应过程中反应物 的结构变化有可能导致不同的反应机理. 2007 年, Rebek 等 ${ }^{[30]}$ 使用均三甲苯形成受体空腔结构研究氨基醇脱水 的反应机理, 由于屏蔽了氨基醇与水分子的接触, 在有 机溶剂下, 他们认为氨基醇通过四元环过渡态脱水是主 要途径. 2013 年, Di Stefano 等 ${ }^{[31]}$ 在有机溶剂中进行了苯
甲醛和丁胺之间可逆缩合的反应动力学研究, 指出溶剂 性质对反应速率有显著影响.

受前面工作的启发, 我们采用 Jørgensen 研究小组 ${ }^{[2]}$ 提出的 $\mathrm{MeO}-\mathrm{PhCH}\left(\mathrm{CH}_{3}\right) \mathrm{CHO}$ 与新型伯胺催化剂 $\mathrm{O}\left(\mathrm{CH}_{2} \mathrm{CH}_{2}\right)_{2} \mathrm{NCH}_{2} \mathrm{CH}(t-\mathrm{Bu}) \mathrm{NH}_{2}$ 作为研究对象, 通过密 度泛函理论计算研究了酸性和二氯甲烷溶剂环境中两 者发生的缩合反应机理，指出邻近氮杂环体系中氮原子 协助氢转移路径的重要性, 为我们研究含邻近氮原子醛 胺体系的醛胺缩合反应机理提供一定的理论参考.

\section{2 结果与讨论}

\section{1 酸性条件下胺与醛反应}

在酸性条件下, 氢质子可以与羰基氧和含邻近氮杂 环胺底物的杂环氮原子结合，根据结合位点的不同可分 为两条反应路径(见图 2 中 Path 1 和 Path 2). 在 Path 1 中, 胺底物 $\mathbf{1}$ 的杂环氮原子首先被质子化, 随后与醛形 成中间体 $4 \mathrm{a}$, 中间体 $4 \mathrm{a}$ 经过构型转化形成 $4 \mathrm{~b}$, 使伯胺 氮上的氢原子通过杂环氮原子转移到羟基形成中间体 6, 最后脱去一分子水得到质子化亚胺 7. 在 Path 2 中, 氢质子先与 2 的羰基氧结合, 再与胺底物形成中间体 $4 c$, 最后脱水得到 7 .

氢质子与胺的氮原子结合生成铵盐降低反应速

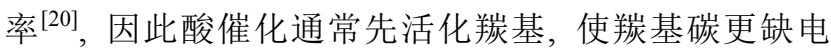
子, 从而加强与伯胺的结合作用. 当反应物胺含有邻近 杂环氮原子时，杂环上的氮原子的碱性强于羰基，所以 氢优先质子化 1 上的杂环氮原子形成 3 . 如图 3 中 Path 1 所示, 该反应步骤自由能变化为 $-46.42 \mathrm{kcal} / \mathrm{mol}$. 随后 在羰基碳和胺上氨基氮形成 $\mathrm{C}-\mathrm{N}$ 键步骤中, 杂环氮上 的氢质子转移到崖基氧上, 形成质子化氨基醇 $4 \mathrm{a}$, 能量 上升 $1.07 \mathrm{kcal} / \mathrm{mol}$. 如图 4 所示, $4 \mathrm{a}$ 中碳氮键长为 1.532 $\AA$, 且 $\mathrm{H}(2)$ 指向 $\mathrm{N}(1)$, 两原子间距为 $1.659 \AA$, 形成一个 弱氢键. 之后胺上的一个氢与杂环氮作用, 使得原本的 羟基被排斥旋转到另一边形成中间体 $\mathbf{4 b}$, 能量升高 3.24 $\mathrm{kcal} / \mathrm{mol}$. 从 $4 \mathrm{~b}$ 到过渡态 $\mathbf{T S} 1$, 氢原子从伯胺转移到碱 性更强的杂环氮原子上, 形成一个五元环过渡态, 反应 能垒为 $0.85 \mathrm{kcal} / \mathrm{mol}$. N(1)- $\mathrm{H}(1)$ 键的键长由 $1.053 \AA$ 伸 长到 $1.242 \AA$, 而 $\mathrm{H}(1)-\mathrm{N}(1)$ 距离则由 $1.846 \AA$ 缩短到 $1.345 \AA$. 随后, $\mathrm{H}(1)-\mathrm{N}(1)$ 距离继续缩短到 $1.036 \AA$, 形 成中间体 $\mathbf{5}$, 能量下降 $7.38 \mathrm{kcal} / \mathrm{mol}$. 然后氢转移到羟基 氧上, 反应位垒为 $13.08 \mathrm{kcal} / \mathrm{mol}$. 从 5 到 $\mathbf{T S 2}, \mathrm{H}(1)$ 一 $\mathrm{O}(1)$ 距离由 $1.878 \AA$ 缩短至过渡态 TS2 的 $1.085 \AA$. $\mathrm{C}(1)-\mathrm{O}(1)$ 键伸长到 $1.851 \AA, \mathrm{C}(1)$ - $\mathrm{N}(2)$ 键长缩短到 $1.329 \AA$. 随着 $\mathrm{C}(1)-\mathrm{O}(1)$ 单键的断裂以及 $\mathrm{C}(1)-\mathrm{N}(2)$ 双 键的形成, 形成水和质子化亚胺复合物 $\mathbf{6}$, 最后脱水生 成质子化亚胺 7.

Path 2 是氢质子先质子化羰基氧形成中间体 $\mathbf{8}$, 然 后与 1 反应形成中间体 $\mathbf{4 c}$. 由于杂环位于 $\mathbf{4 c}$ 另一平面, 杂环氮原子无法作为氢转移桥, 氢质子与邻近羟基氧形 


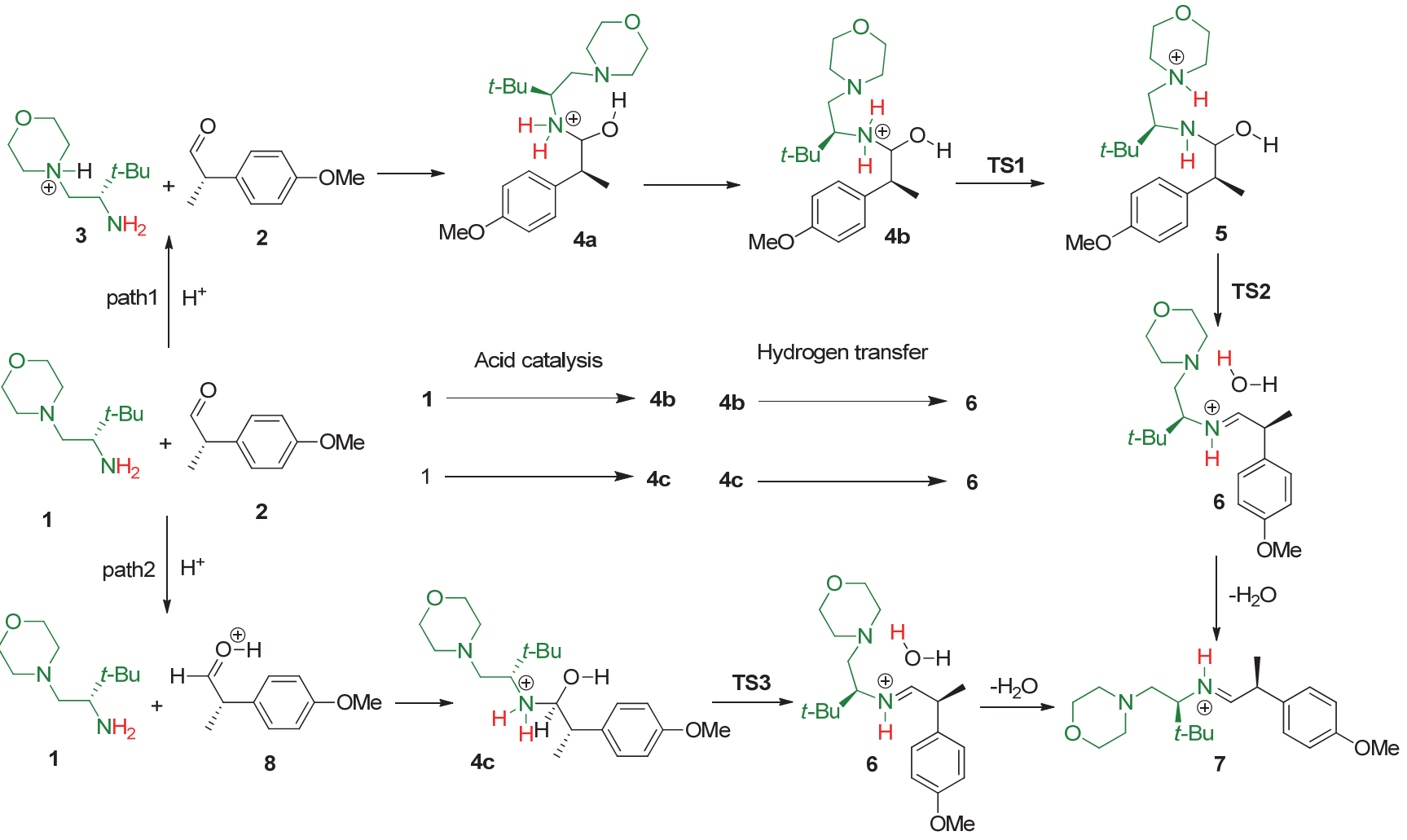

图 2 酸性条件下带邻近氮杂环胺与醛反应的反应路径示意图

Figure 2 The reaction pathways of the aldimine condensation of amine with nitrogenous heterocycle and aldehyde in acidic conditions

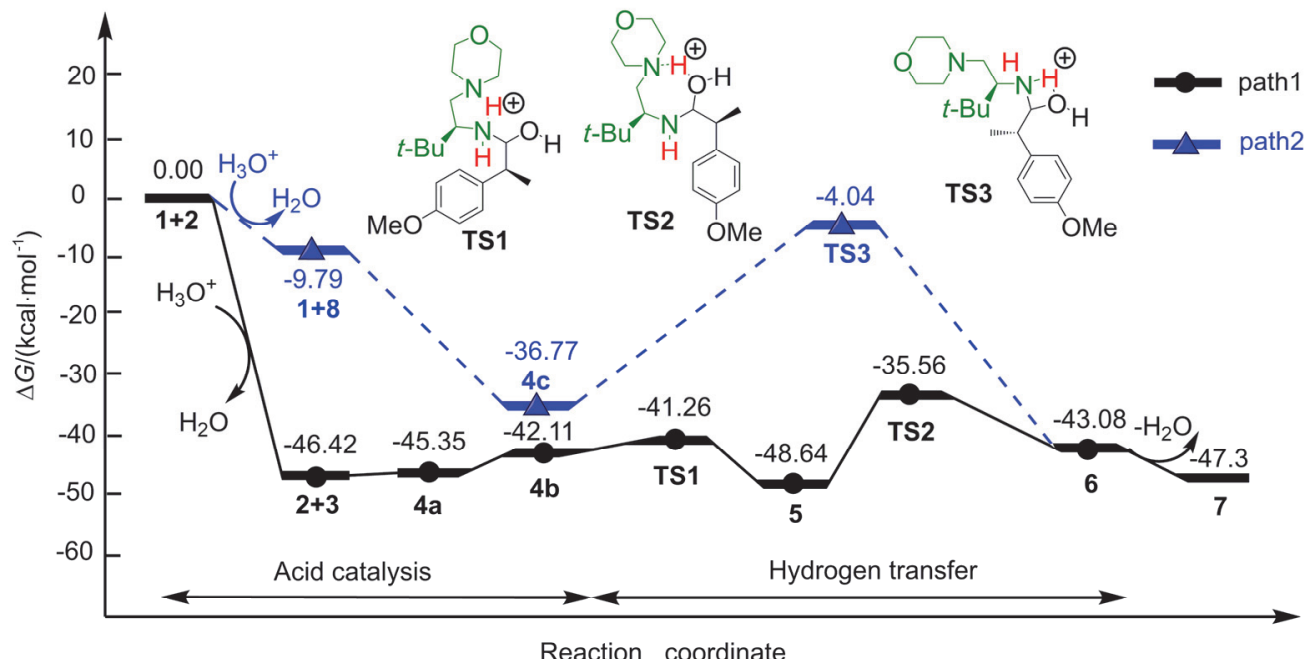

图 3 酸性条件下带邻近氮杂环胺与醛反应的自由能示意图(能量: $\mathrm{kcal} / \mathrm{mol}$ )

Figure 3 Free energy profiles for the aldimine condensation of amine with nitrogenous heterocycle and aldehyde in acidic conditions (all energies are denoted in $\mathrm{kcal} / \mathrm{mol}$ )

成四元环过渡态 TS3 生成中间体 6 , 这一步反应能垒为 $32.73 \mathrm{kcal} / \mathrm{mol}$. 在此过程中 $\mathrm{O}(1)-\mathrm{H}(1)$ 距离由 $2.212 \AA$ 缩短至 $1.150 \AA . \mathrm{N}(2)-\mathrm{H}(1)$ 键的键长伸长至 $1.432 \AA$, $\angle \mathrm{N}(2)-\mathrm{C}(1)-\mathrm{O}(1)$ 由 $4 \mathbf{c}$ 的 $105.60^{\circ}$ 变为 $\mathbf{T S 3}$ 的 $94.36^{\circ}$, 环张力的改变可能是此步骤能垒较高的原因. 最后中间 体 6 失去水分子得到质子化的亚胺 7.

比较 path 1 和 path 2 , 无论从动力学角度还是热力
学角度均表明 path 1 中氢质子先与底物胺 $\mathbf{1}$ 上邻近杂环 氮原子结合生成 3 是优势反应路径. 而 path 2 中直接氢 转移脱水的反应能垒远高于 path 1 中邻近杂环氮协助分 步氢转移路径, 这些结果指出了在醛胺缩合反应邻近氮 原子在氢转移脱水分子生成亚胺反应中的重要作用. 同 时, 我们也计算了水分子协助氢转移反应过程. 但因为 临近杂环, 在当前计算级别下找不到水分子协助脱水形 


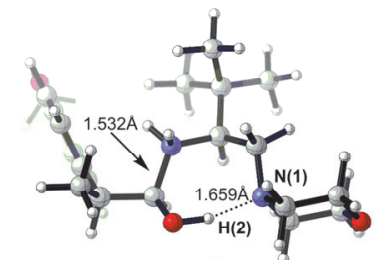

4a
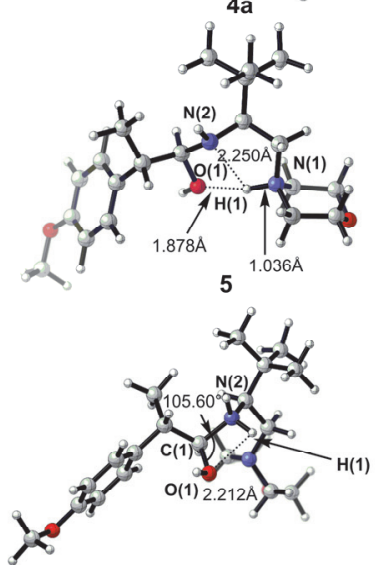

$4 c$

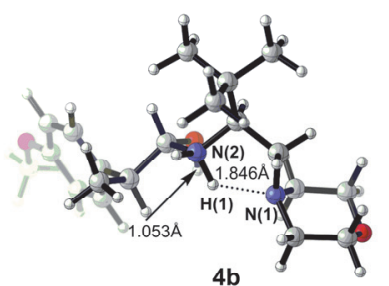

4b

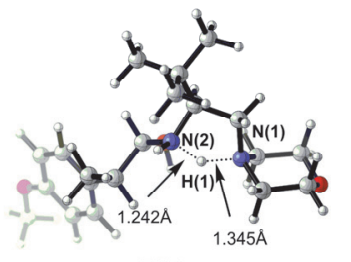

TS1
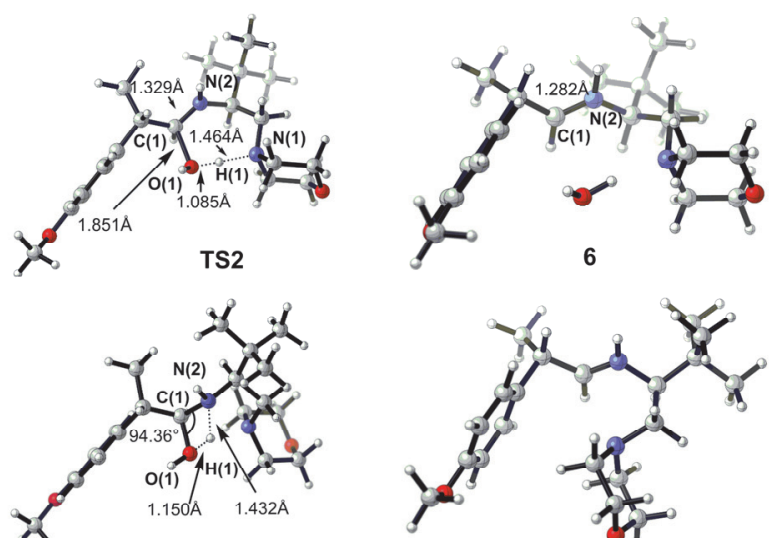

TS3

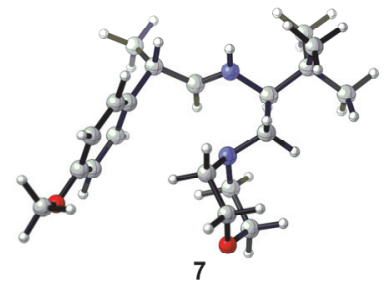

图 4 带邻近氮杂环胺与醛缩合反应计算的关键驻点几何结构

Figure 4 The optimized geometries of stationary points along the condensation of amine with nitrogenous heterocycle and aldehyde in acidic conditions

成质子化亚胺，反而是在羟基接受水分子传递氢的同 时, 本身的氢也重新回到杂环氮上, 即 $\mathbf{4 a}$ 中羟基氢与杂 环氮形成的是一个低能垒氢键, 周围环境的变化导致氢 重新转移回杂环氮上, 使得整体不是一个 $4 \mathrm{c}$ 到 6 的过 程, 而是一个 $4 \mathrm{a}$ 到 5 的过程. 计算得到的反应位垒分别 为 11.39 和 $12.52 \mathrm{kcal} / \mathrm{mol}$ (详见 SI 中 Fig. S4). 这进一步 说明了含有邻近氮杂环胺醛缩合反应体系中邻近氮原 子辅助氢转移的重要作用.

\section{2 邻近杂原子调变效应研究}

为了研究邻近杂原子调变效应, 我们将胺邻近杂环

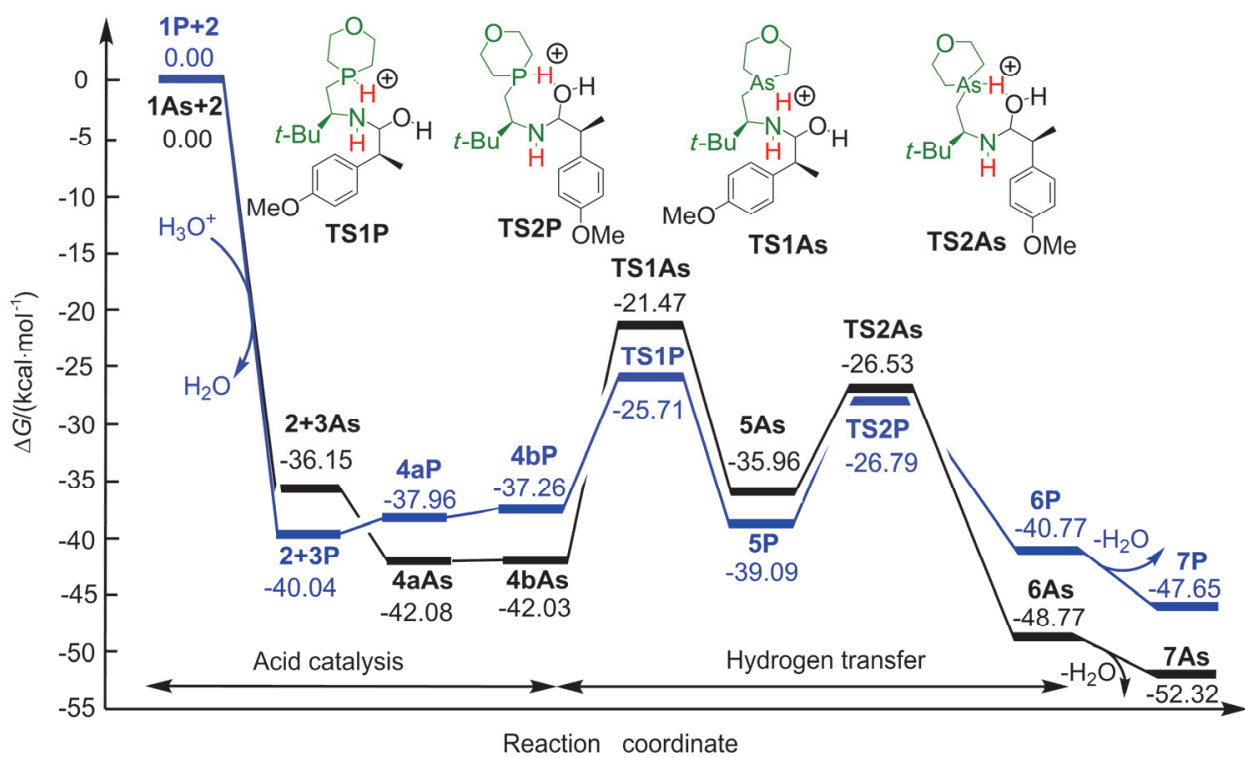

图 5 伯胺的杂原子为 $\mathrm{P}, \mathrm{As}$ 时的反应的自由能示意图(能量: $\mathrm{kcal} / \mathrm{mol}$ )

Figure 5 Free energy profiles in the reactions when heteroatom of primary amine is $\mathrm{P}$ and As (all energies are denoted in $\mathrm{kcal} / \mathrm{mol}$ ) $\mathrm{P}, \mathrm{As}$ 原子, 此过程与前面讨论类似, 同样是一个热力学 稳定的过程. 相对于 3 能量下降了 $46.42 \mathrm{kcal} / \mathrm{mol}$, 当底 物 $\mathrm{N}$ 调变为 $\mathrm{P}$ 和 $\mathrm{As}$ 原子后质子化中间体能量分别降低 40.04 和 $36.15 \mathrm{kcal} / \mathrm{mol}$. 热力学稳定性依次降低, 这可 能是因为 $\mathrm{P}, \mathrm{As}$ 原子的碱性不如 $\mathrm{N}$ 原子. 之后 $3 \mathrm{P}$ 与醛 2 
表 1 关键驻点几何结构部分键的键级

Table 1 Bond orders of the key optimized geometries of stationary points

\begin{tabular}{lllll}
\hline Stationary points & Chemical bond & Mayer & Wiberg & $\begin{array}{l}\text { Relative free } \\
\text { energy } \\
G /\left(\mathrm{kcal}^{\circ} \mathrm{mol}^{-1}\right)\end{array}$ \\
\hline 4b & $\mathrm{H}(1)-\mathrm{N}(2)$ & 0.798 & 0.863 & 0.00 \\
4bP & $\mathrm{H}(1)-\mathrm{N}(2)$ & 0.861 & 0.917 & 0.00 \\
4bAs & $\mathrm{H}(1)-\mathrm{N}(2)$ & 0.864 & 0.920 & 0.00 \\
TS1 & $\mathrm{H}(1)-\mathrm{N}(2)$ & 0.526 & 0.629 & 0.85 \\
TS1P & $\mathrm{H}(1)-\mathrm{N}(2)$ & 0.312 & 0.396 & 11.05 \\
TS1As & $\mathrm{H}(1)-\mathrm{N}(2)$ & 0.279 & 0.322 & 20.56 \\
$\mathbf{5}$ & $\mathrm{H}(1)-\mathrm{N}(1)$ & 0.812 & 0.882 & 0.00 \\
$\mathbf{5 P}$ & $\mathrm{H}(1)-\mathrm{P}(1)$ & 0.913 & 0.930 & 0.00 \\
$\mathbf{5 A s}$ & $\mathrm{H}(1)-\mathrm{As}(1)$ & 0.926 & 0.955 & 0.00 \\
TS2 & $\mathrm{H}(1)-\mathrm{N}(1)$ & 0.286 & 0.427 & 13.08 \\
TS2P & $\mathrm{H}(1)-\mathrm{P}(1)$ & 0.500 & 0.623 & 12.30 \\
TS2As & $\mathrm{H}(1)-\mathrm{As}(1)$ & 0.547 & 0.672 & 9.43 \\
\hline
\end{tabular}

形成 $4 \mathrm{aP}$ 时，能量上升了 $2.08 \mathrm{kcal} / \mathrm{mol}$. 而 $\mathbf{3 A s}$ 与 2 形 成 $4 \mathrm{aAs}$ 时, 能量下降了 $5.93 \mathrm{kcal} / \mathrm{mol}$, 之后通过构型变 化, 伯胺的一个氢指向杂原子, $4 \mathrm{bP}, 4 \mathrm{bAs}$ 能量分别上升 0.70 和 $0.05 \mathrm{kcal} / \mathrm{mol}$.

第一个氢转移反应位垒在调变体系 P 和 As 中提高 到 11.55 和 $20.56 \mathrm{kcal} / \mathrm{mol}$. 我们计算了此步反应中关键 原子的 Mayer 及 Wiberg 键级, 结果表明, Mayer 及 Wiberg 键级差别较小, 这也为数据的对比提供了可靠 的支持. $4 \mathrm{~b}$ 的 $\mathrm{H}(1)-\mathrm{N}(2)$ 的 Mayer 键级为 0.798 , 而 $4 \mathrm{bP}$ 中为 $0.861, \mathbf{4 b A s}$ 为 0.864 , 这是因为 $\mathbf{4 b}$ 中 $\mathrm{H}(1)$ 除了与 $\mathrm{N}(2)$ 成键以外还与 $\mathrm{N}(1)$ 之间存在一个氢键削弱了 $\mathrm{H}(1)-\mathrm{N}(2)$ 键. 而相应的 $\mathrm{P}$, As 原子因为过大而远离 $\mathrm{H}(1)$ ，从而 $\mathrm{H}(1)$ 与 $\mathrm{N}(2)$ 之间有更好的成键作用. 而从 $\mathbf{4 b}$ 到 TS1, H(1)- $\mathrm{N}(2)$ 的 Mayer 键级降低了 0.272, 调变后 分别是 0.549 和 0.585 , Wiberg 键级则分别下降 0.234 , 0.521 和 0.598 . 在第二个氢转移步骤中, $\mathrm{H}(1)$ 向 $\mathrm{O}(1)$ 转 移, TS2, TS2P 和 TS2As 中的 $\mathrm{H}(1)-\mathrm{X}(1)(\mathrm{X}=\mathrm{N}, \mathrm{P}, \mathrm{As})$ 与 5, 5P, 5As 相比, Mayer 键级分别下降了 0.526, 0.413, 0.379. Wiberg 键级则降低 $0.455,0.307,0.283$. 与反应能 垒呈现同样的变化趋势. 最后 $6 \mathrm{P}, 6 \mathrm{As}$ 脱去水分子, 得 到产物 7P 和 7As.

通过杂原子调变, 整个反应的速度控制步骤发生了 变化. $\mathrm{N}$ 原子体系的速控步骤是第二个氢转移步骤, $\mathrm{P}$ 原 子体系两个氢转移步骤能垒相近, As 原子体系反应的速 控步骤变化为第一个氢转移步骤. 如图 6 所示, 键级的 降低和反应能垒的变化趋势一致. 伯胺氮上的氢经过 $\mathrm{TS} 1$ 转移到杂环氮原子上, $\mathrm{H}(1)-\mathrm{N}(2)$ 键的键级变化在 $\mathrm{N} 、 \mathrm{P}$ 和 As 的体系中逐渐增大, 表明 $\mathrm{H}(1)-\mathrm{N}(2)$ 随着杂 原子的调变越来越难断裂, 氢迁移难度逐渐加大, 第一
个氢转移步骤能垒逐渐提高. 随后, 杂环原子上氢经过 TS2 转移到羟基上, $\mathrm{H}(1)-\mathrm{X}(1)(\mathrm{X}=\mathrm{N}, \mathrm{P}, \mathrm{As})$ 的键级变 化在 $\mathrm{N} 、 \mathrm{P}$ 和 $\mathrm{As}$ 的体系中逐渐减小, 表明随着杂原子的 调变 $\mathrm{H}(1)-\mathrm{X}(1)$ 键越来越容易断裂, 氢迁移变得相对容 易, 第二步氢转移能垒逐渐降低. 杂原子的电荷随着原 子调变逐渐增大(见 SI 中图 S11), 碱性减弱, 造成第一 个氢转移能垒增加, 第二个氢转移能垒降低, 这与键级 变化分析一致. 通过调变伯胺杂环上的氮原子, 展现了 不同电子密度的杂原子对反应活性的影响，随着杂原子 电子密度的降低, 反应能垒逐渐提高. 进一步肯定了邻 近杂原子作为氢转移媒介的重要作用.

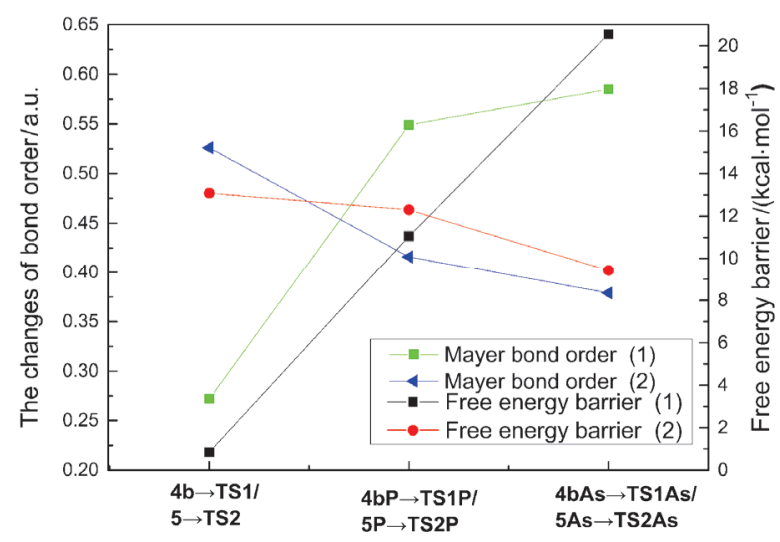

图 6 键级的变化与反应能垒示意图

Figure 6 The profiles of relationship between the change of bond order and energy barrier

\section{3 邻近氮原子位置调变效应研究}

为了进一步研究邻近氮原子作为氢转移媒介对反 应机理的影响. 我们对氮原子所处位置进行了调变. 各 驻点的名称标注与上述研究反应路径各驻点名称对应 (图 7). 为简洁起见, 我们选取 1-己胺为反应底物, 将其 2, 3, 4, 5 号位的碳原子分别调变成氮原子与乙醛 2ald 反 应, 并以质子化胺与乙醛单独存在的能量之和为零点. 图中只给出氮原子处于 3 号位时的结构(其他见 SI). 如 图所示, 质子化胺与乙醛结合成 $\mathbf{4 a}_{\mathrm{Ni}}$ 时除 $\mathbf{4 a}_{\mathrm{N} 3}$ 之外其他 能量都有明显上升, 这是因为它们都是从乙醛 $s i$ 面进 攻, 甲基和环存在空间排斥. 之后氮原子处于 3, 4, 5 号 位上能垒都较低, 反应的能量跨度分别为 $11.46,14.41$ 和 $13.94 \mathrm{kcal} / \mathrm{mol}$. 可以看出, 当氮原子处于 3 号位时醛 胺缩合反应最好. 有趣的是, 当氮原子处于 2 号位时不 仅后一步能垒提高到 $18.01 \mathrm{kcal} / \mathrm{mol}$, 而且整个反应的 速控步骤变成了第一个氢转移步骤, 能量跨度高达 $28.59 \mathrm{kcal} / \mathrm{mol}$, 这是因为对于过渡态 $\mathbf{T S 1}_{\mathrm{N} 2}$ 而言，它经 过的同样是一个四元环过渡态, 这和氢直接转移到羟基 上脱水是相似的, 这证实了过渡态结构中的环张力是导 致醛胺缩合直接脱水难度大的原因. 通过对氮原子所处 位置的调变, 我们进一步确定了环张力是导致醛胺缩合 直接脱水的能垒高的原因. 因此, 在邻近存在合适的质 子转移媒介对醛胺缩合是极为有利的. 


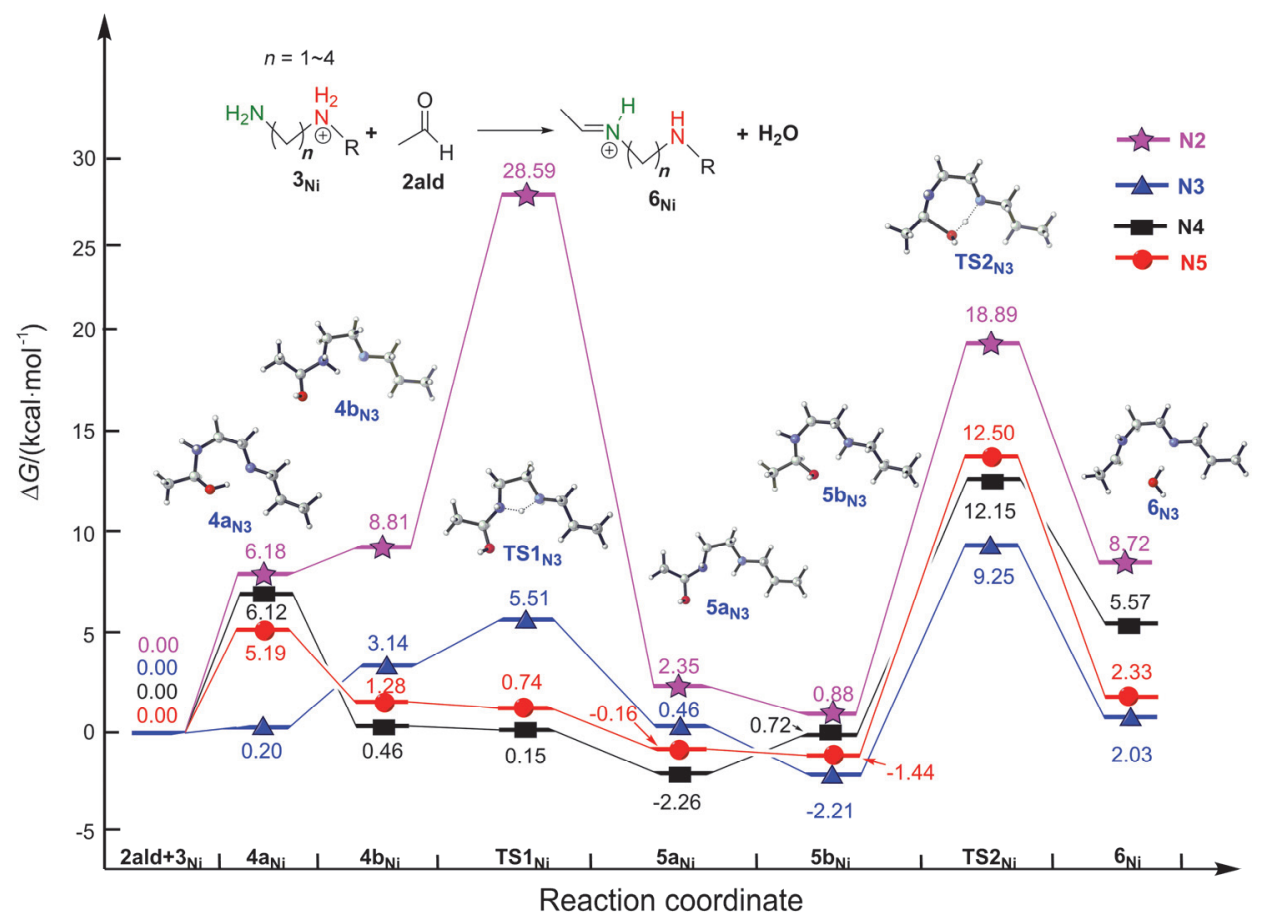

图 7 邻近氮原子位置调变时反应的自由能示意图

Figure 7 Free energy profiles in the reactions when the position of adjacent nitrogen atom is modulated

\section{3 结论}

在 $\omega \mathrm{B} 97 \mathrm{X}-\mathrm{D} / 6-31++\mathrm{G}(\mathrm{d}, \mathrm{p})$ 水平下，对含氮杂环的 伯胺与醛在酸性条件下有机溶剂二氯甲烷作为溶剂时 的反应机理进行了研究. 研究结果表明, 和通常的酸催 化醛胺缩合活化羰基相比, 杂环氮是个更好的反应位 点, 其碱性远优于羰基氧并且可以避免形成环张力较大 的四元环过渡态, 每一步的氢转移都可以借助杂环氮作 媒介, 使反应能垒大大降低. 氮原子体系的酫胺缩合反 应的速控步骤是第二个氢转移步骤, 反应能垒为 13.08 $\mathrm{kcal} / \mathrm{mol}$. 同时, 对伯胺杂环上的氮原子调变为 $P$ 原子和 As 原子的调变研究表明, 随着杂原子电子密度的降低, 反应能垒逐渐提高. 对底物中- $\mathrm{NH}_{2}$ 基团邻近氮原子位 置的调变效应研究表明, 当氮原子处于 3 号位时醛胺缩 合反应最好. 上述研究揭示了邻近氮原子作为氢转移媒 介的重要作用, 这为深刻理解和认识伯胺与醛的酫胺缩 合反应机理提供了新的思路.

\section{4 计算方法}

本课题中所有中间体和过渡态的结构均采用密度 泛函理论(DFT) $\omega$ B $97 \mathrm{X}-\mathrm{D}$ 方法 ${ }^{[32]}$, 所有原子均采用 $6-31++\mathrm{G}(\mathrm{d}, \mathrm{p})$ 基组. 以二氯甲烷为溶剂进行计算, 采 用了 Truhlar 等 ${ }^{[33]}$ 提出的 SMD 溶剂化模型. 在本课题中 所有能量均为采用 $\omega \mathrm{B} 97 \mathrm{X}-\mathrm{D}$ 方法以二氯甲烷为溶剂计 算得出的吉布斯自由能, 其中氢质子的溶剂化自由能用 $\mathrm{H}_{3} \mathrm{O}^{+}$和 $\mathrm{H}_{2} \mathrm{O}$ 能量之差近似. 通过对反应过程中的驻点 进行振动频率计算, 确认了所有的稳定点均无虚频, 全
部过渡态有且仅有一个负本征值. 对所有过渡态结构做 了内禀反应坐标(IRC)分析，证实了过渡态可以很好地 与左右中间体连接 ${ }^{[34]}$. 所有分子没有进行简化处理, 均 进行全模型优化，以求与真实反应条件相接近. 键级分 析 ${ }^{[35,36]}$ 是在 6-311G(d,p)级别下通过 Multiwfn 程序 ${ }^{[3]}$ 辅 $^{-}$ 助完成. 本理论研究所使用的软件为 Gaussian 09 程序 包 ${ }^{[38]}$. 文中呈现分子结构所用的软件是 CYLView ${ }^{[39]}$.

\section{References}

[1] Schiff, H. Justus Liebigs Ann. Chem. 1864, 131, 118.

[2] Leth, L. A.; Naesborg, L.; Reyes-Rodriguez, G. J.; Tobiesen, H. N.; Iversen, M. V.; Jorgensen, K. A. J. Am. Chem. Soc. 2018, 140, 12687.

[3] Rezayee, N. M.; Lauridsen, V. H.; Naesborg, L.; Nguyen, T. V. Q.; Tobiesen, H. N.; Jorgensen, K. A. Chem. Sci. 2019, 10, 3586.

[4] Liu, Y.; Yue, X.; Luo, C.; Zhang, L.; Lei, M. Energy Environ. Mater. 2019, 2, 292.

[5] Stana, A.; Enache, A.; Vodnar, D. C.; Nastasa, C.; Benedec, D.; Ionut, I.; Login, C.; Marc, G.; Oniga, O.; Tiperciuc, B. Molecules 2016, 21, 1595 .

[6] Hong, M.; Min, J.; Wang, S. Chin. J. Org. Chem. 2018, 38, 1907 (in Chinese). (洪梅, 闵洁, 王石发, 有机化学, 2018, 38, 1907.)

[7] Li, Y.; Jia, F.; Ma, L.; Li, Z. Acta Chim. Sinica 2015, 73, 1311 (in Chinese). (李远明, 贾凡, 马丽娜, 李志平, 化学学报, 2015, 73, 1311.)

[8] Hu, S.-B.; Chen, M.-W.; Zhai, X.-Y.; Zhou, Y.-G. Acta Chim. Sinica 2018, 76, 103 (in Chinese). (胡书博, 陈木旺, 翟小勇, 周永贵, 化 学学报, 2018, 76, 103.)

[9] Wang, H.; Huang, L. Chin. J. Org. Chem. 2019, 39, 883 (in Chinese). (王辉, 黄龙江, 有机化学, 2019, 39, 883.)

[10] Xiao, M.; Yue, X.; Xu, R.; Tang, W.; Xue, D.; Li, C.; Lei, M.; Xiao, J.; Wang, C. Angew. Chem., Int. Ed. 2019, 58, 10528.

[11] Santerre, G. M.; Hansrote, C. J.; Crowell, T. I. J. Am. Chem. Soc. 1958, 80, 1254.

[12] Martin, R. B. J. Phys. Chem. 1964, 68, 1369.

[13] Makela, M. J.; Korpela, T. K. Chem. Soc. Rev. 1983, 12, 309. 
[14] Jencks, W. P. Prog. Phys. Org. Chem. 1964, 2, 63

[15] Hine, J.; Via, F. A.; Gotkis, J. K.; Craig, J. C. J. Am. Chem. Soc. 1970, 92, 5186.

[16] Sayer, J. M.; Pinsky, B.; Schonbrunn, A.; Washtien, W. J. Am. Chem. Soc. 1974, 96, 7998.

[17] Rosenberg, S.; Silver, S. M.; Sayer, J. M.; Jencks, W. P. J. Am. Chem. Soc. 1974, 96, 7986.

[18] Williams, I. H. J. Am. Chem. Soc. 1987, 109, 6299.

[19] Hall, N. E.; Smith, B. J. J. Phys. Chem. A 1998, 102, 4930.

[20] Jencks, W. P. J. Am. Chem. Soc. 1959, 81, 475.

[21] Jencks, W. P. Acc. Chem. Res. 1976, 9, 425.

[22] Sayer, J. M.; Jencks, W. P. J. Am. Chem. Soc. 1973, 95, 5637.

[23] Ding, Y. Q.; Cui, Y. Z.; Li, T. D. J. Phys. Chem. A 2015, 119, 4252.

[24] Salva, A.; Donoso, J.; Frau, J.; Muñoz, F. J. Phys. Chem. A 2003, $107,9409$.

[25] Ortega-Castro, J.; Adrover, M.; Frau, J.; Salva, A.; Donoso, J.; Muñoz, F. J. Phys. Chem. A 2010, 114, 4634.

[26] Casasnovas, R.; Salvà, A.; Frau, J.; Donoso, J.; Muñoz, F. Chem. Phys. 2009, 355, 149.

[27] Solis-Calero, C.; Ortega-Castro, J.; Muñoz, F. J. Phys. Chem. B 2010, 114, 15879.

[28] Kirmizialtin, S.; Yildiz, B. S.; Yildiz, I. J. Phys. Org. Chem. 2017, 30,1 .

[29] Ciaccia, M.; Di Stefano, S. Org. Biomol. Chem. 2015, 13, 646

[30] Hooley, R. J.; Iwasawa, T.; Rebek, J., Jr. J. Am. Chem. Soc. 2007, 129, 15330.

[31] Ciaccia, M.; Cacciapaglia, R.; Mencarelli, P.; Mandolini, L.; Di Stefano, S. Chem. Sci. 2013, 4, 2253.
[32] Chai, J.-D.; Head-Gordon, M. Phys. Chem. Chem. Phys. 2008, 10, 6615.

[33] Marenich, A. V.; Cramer, C. J.; Truhlar, D. G. J. Phys. Chem. B 2009, 113, 6378 .

[34] Hratchian, H. P.; Schlegel, H. B. J. Chem. Phys. 2004, 120, 9918.

[35] Mayer, I. Chem. Phys. Lett. 1983, 97, 270.

[36] Wiberg, K. B. Tetrahedron 1968, 24, 1083

[37] Lu, T.; Chen, F. J. Comput. Chem. 2012, 33, 580.

[38] Frisch, M. J.; Trucks, G. W.; Schlegel, H. B.; Scuseria, G. E.; Robb M. A.; Cheeseman, J. R.; Scalmani, G.; Barone, V.; Mennucci, B.; Petersson, G. A.; Nakatsuji, H.; Caricato, M.; Li, X.; Hratchian, H. P.; Izmaylov, A. F.; Bloino, J.; Zheng, G.; Sonnenberg, J. L.; Hada, M.; Ehara, M.; Toyota, K.; Fukuda, R.; Hasegawa, J.; Ishida, M.; Nakajima, T.; Honda, Y.; Kitao, O.; Nakai, H.; Vreven, T.; Montgomery Jr., J. A.; Peralta, J. E.; Ogliaro, F.; Bearpark, M.; Heyd, J. J.; Brothers, E.; Kudin, K. N.; Staroverov, V. N.; Keith, T.; Kobayashi, R.; Normand, J.; Raghavachari, K.; Rendell, A.; Burant, J. C.; Iyengar, S. S.; Tomasi, J.; Cossi, M.; Rega, N.; Millam, J. M.; Klene, M.; Knox, J. E.; Cross, J. B.; Bakken, V.; Adamo, C.; Jaramillo, J.; Gomperts, R.; Stratmann, R. E.; Yazyev, O.; Austin, A. J.; Cammi, R.; Pomelli, C.; Ochterski, J. W.; Martin, R. L.; Morokuma, K.; Zakrzewski, V. G.; Voth, G. A.; Salvador, P.; Dannenberg, J. J.; Dapprich, S.; Daniels, A. D.; Farkas, O.; Foresman, J. B.; Ortiz, J. V.; Cioslowski, J.; Fox, D. J. Gaussian 09, Revision B. 01. Gaussian, Inc., Wallingford, $\mathbf{2 0 1 0 .}$

[39] Legault, C. Y. CYLview, 1.0b, Université de Sherbrooke, 2009, http://www.cylview.org. 